This item was submitted to Loughborough's Research Repository by the author.

Items in Figshare are protected by copyright, with all rights reserved, unless otherwise indicated.

\title{
Prototype system for knowledge problem definition
}

PLEASE CITE THE PUBLISHED VERSION

PUBLISHER

(C) ASCE

VERSION

AM (Accepted Manuscript)

LICENCE

CC BY-NC-ND 4.0

REPOSITORY RECORD

Al-Ghassani, Ahmed M., John M. Kamara, Chimay J. Anumba, and Patricia M. Carrillo. 2019. "Prototype System for Knowledge Problem Definition". figshare. https://hdl.handle.net/2134/4188. 
This item was submitted to Loughborough's Institutional Repository (https://dspace.lboro.ac.uk/) by the author and is made available under the following Creative Commons Licence conditions.

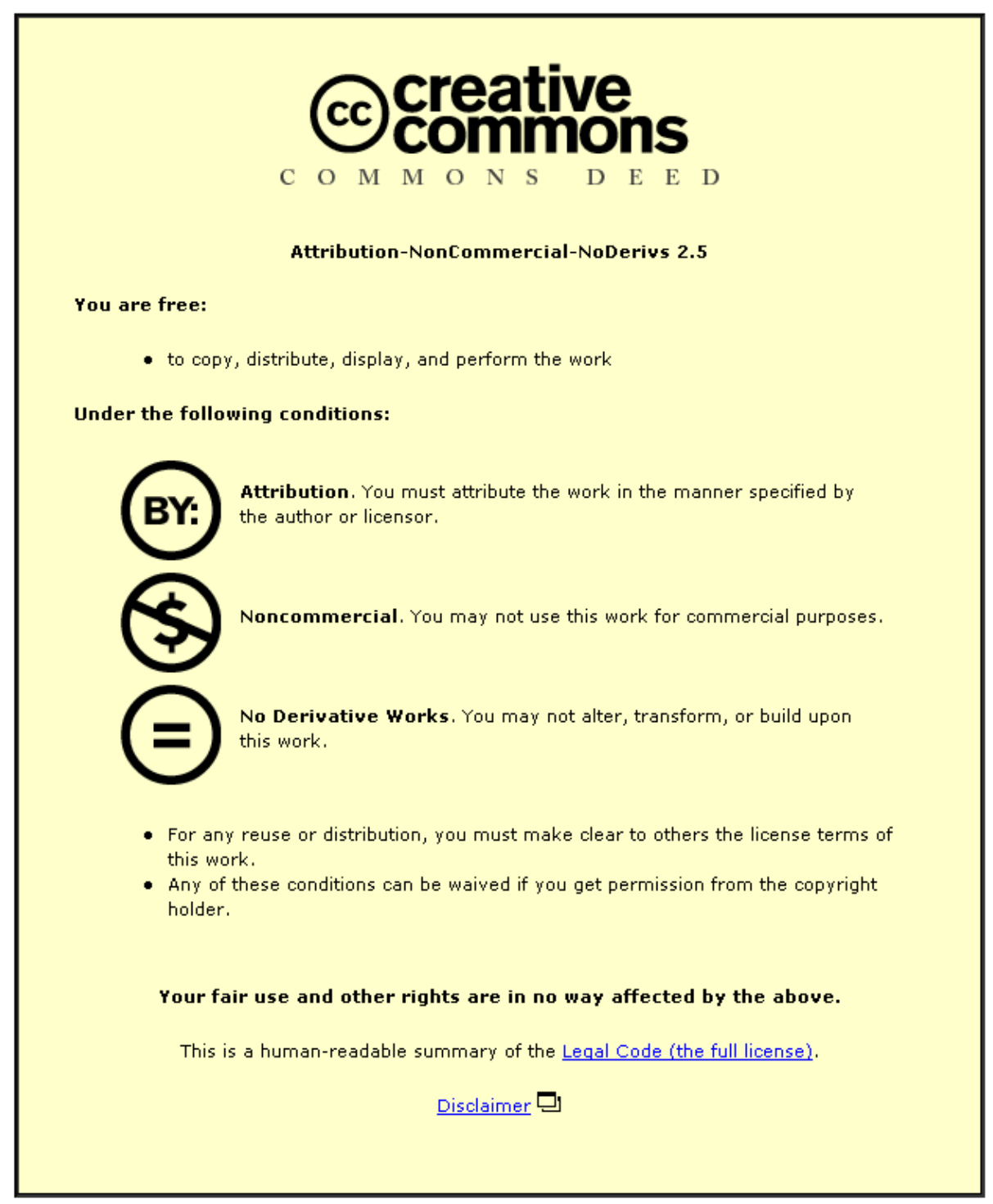

For the full text of this licence, please go to: http://creativecommons.org/licenses/by-nc-nd/2.5/ 


\title{
A Prototype System for Knowledge Problem Definition
}

\author{
Ahmed M. Al-Ghassani ${ }^{1}$, Member, ASCE; John M. Kamara², Member, ASCE; Chimay J. \\ Anumba $^{3}$, Member, ASCE and Patricia M. Carrillo $^{4}$
}

\begin{abstract}
Attitudes to knowledge management (KM) have changed considerably as organizations are now realizing its benefits. Implementation, however, has been facing serious difficulties attributed to either not being able to anticipate the barriers when planning $\mathrm{KM}$ strategies or to using inappropriate methods and tools for implementation. These difficulties are more critical in construction due to the fragmented nature of the industry. This paper suggests that proper definition of a KM problem at the early stages of developing the KM initiatives will result in better control over the KM barriers. A methodology for identifying KM problems within a business context is then introduced. The methodology is encapsulated into a prototype software system, which facilitates its deployment in organizations and provides online help facilities. The methodology, development, operation, and evaluation of the prototype are described. The paper concludes that the prototype offers considerable potential for delivering a clarified KM problem and a distilled set of issues for an organization to address. This represents a significant first step in any $\mathrm{KM}$ initiative.
\end{abstract}

Key Words: information technology, knowledge, management, organizations

\footnotetext{
${ }_{1}^{1}$ Assistant Dean for Academic Affairs, Al-Musanna College of Technology, P.O. Box 191, P.C. 314, AlMuluddah, Oman, Email: ahmed@alghassani.com

${ }^{2}$ Lecturer, School of Architecture, Planning and Landscape, University of Newcastle upon Tyne, 3-4

Claremont Terrace, Newcastle upon Tyne NE2 4AE, UK., Tel +44 (0) 191222 8619, Fax +44 (0) 191222 8230, Email: j.m.kamara@ncl.ac.uk

${ }^{3}$ Professor, Department of Civil and Building Engineering, Loughborough University, Loughborough, Leicestershire, LE11 3TU, UK., Tel +44 (0)1509 222615, Fax +44 (0)1509 222982, Email:

c.j.anumba@lboro.ac.uk

${ }^{4}$ Senior Lecturer, Department of Civil and Building Engineering, Loughborough University, Loughborough, Leicestershire, LE11 3TU, UK., Tel +44 (0)1509 222634, Fax +44 (0)1509 223941, Email:

p.m.carrillo@lboro.ac.uk
} 


\section{INTRODUCTION}

Knowledge relates to data and information and this relationship needs to be understood first. Data can be described as discrete facts about events which when processed and given relevant associations and patterns become information (Blumentritt and Johnston 1999). Knowledge is therefore information with context that provides the basis for actions and decision-making (Kanter 1999).

There are several definitions of KM. It can be defined from a process perspective, an outcome perspective, or a combination. An example of a process perspective definition sees KM as a process of controlling the creation, dissemination, and utilization of knowledge (Newman 1991; Kazi et al. 1999). Another considers KM as the “... identification, optimization, and active management of intellectual assets, either in the form of explicit knowledge held in artifacts or as tacit knowledge possessed by individuals or communities to hold, share, and grow the tacit knowledge (Snowden 1998). The outcome perspective, on the other hand, focuses on the benefits that an organization gets from managing its knowledge. An example is a definition by Kanter (1999), who sees KM to be concerned with the way an organization gains competitive advantage and builds an innovative and successful organization. Another example considers KM as the "management of organizational knowledge for creating business value and generating competitive advantage" (Tiwana 2000). A third example defines KM as "the ability to create and retain greater value from core business competencies" (Klasson 1999). A combined definition describes both the process by which knowledge is managed and the outcome that is likely to result. Tiwana (2000), for example states that: "Knowledge management enables the creation, communication, and application of knowledge of all kinds to achieve business goals". Another definition by Scarborough et al (1999) states that KM is any process or practice of creating, acquiring, capturing, sharing and using knowledge, wherever it resides, to enhance learning and performance in organizations. Regardless of the different perspectives for defining $\mathrm{KM}$, all 
definitions focus on the fact that knowledge is a valuable asset that must be managed, and that $\mathrm{KM}$ is important to provide strategies to retain knowledge and to improve performance.

The concept of KM is better understood when compared to other concepts such as the organization of work, learning organization, the management of people, sources of wealth creation in contemporary society, the emergence of the information age, organizational learning, the strategic management of core competencies, the management of knowledge-intensive firms, the value of intellectual capital and the management of Research and Development (Scarborough et al. 1999). Figure 1 illustrates one way in which these concepts can relate to one another. For example, the information age is more broadly focused and affects all firms while the management of Research and Development is more narrowly focused and is relevant to a limited number of firms. On the other hand, $\mathrm{KM}$ is more narrowly focused on the ways in which firms facing highly turbulent environments can mobilize their knowledge-base (or knowledge assets) in order to ensure continuous innovation in projects (Scarborough et al. 1999).

<Insert Figure 1 here $>$

This paper presents a methodology developed for identifying KM problems, within a business context, for construction and manufacturing organizations. It then describes the development, operation, and evaluation of a prototype system produced to encapsulate this methodology. The paper first reviews current KM practices. 


\section{CURRENT KM PRACTICES}

\subsection{Overview of research surveys}

Knowledge Management $(\mathrm{KM})$ is making its mark on organizations of all sizes and in all sectors. Surveys show that attitude to KM has changed since 1998 (KPMG 1998). This change in attitude was caused by several reasons, mainly the successful implementation of KM in some organizations and the publishing of two important books on KM by Nonaka and Takeuchi (1995) and Davenport and Prusak (1998). A survey by the Information Systems Research Center of Cranfield School of Management in September 1997 found that almost one third of respondents thought that KM was just a passing fad (KPMG 1998). Surveys after 1998 show that there is a strong belief in the benefits of KM and that it is no longer seen as a passing fad (TFPL 1999; Gottschalk 1999; Robinson et al. 2001). The results of a number of surveys that investigated the awareness of $\mathrm{KM}$ and the width at which it is implemented are discussed below.

A survey of leading UK organizations representing different industry sectors with turnover exceeding £200 M (\$360 M US) a year was undertaken by KPMG Management Consulting (KPMG 1998). The results of the 100 respondents show that KM is not seen as a fad any more but increasingly taken seriously. This was confirmed by $43 \%$ of the respondents who considered their organizations to have KM initiatives in place. The survey also shows that the awareness of $\mathrm{KM}$ increases with the size of organization. It also informs that some organizations implementing KM have already seen real benefits while others had difficulties.

Another survey by TFPL Ltd covered 500 organizations implementing KM or equivalent initiatives from all business sectors around the world (TFPL 1999). Results show that users believe that the concept of KM relies on other concepts such as succeeding in business and enabling organizations to meet their corporate objectives. Of the 80 respondents, $29 \%$ had corporate-wide KM programs and 18\% were planning a corporate-wide KM program. $50 \%$ had 
no corporate-wide $\mathrm{KM}$, but of them, $42 \%$ had another corporate program with similar objectives. The survey concludes that the level of interest in $\mathrm{KM}$ and the number of organizations implementing its initiatives was growing exponentially. Moreover, many chief executives placed KM as second on their list of 'must-dos' after globalization. The survey also shows that most of the KM literature before 1998 concentrated on selling the concept of KM using examples of a few innovative adopters and that much of the focus was on demonstrating the value of intellectual capital, the leveraging of knowledge for competitive advantage, structural issues that underpin $\mathrm{KM}$, and that $\mathrm{KM}$ is mostly about people and culture.

Other surveys that focused on particular industry sectors show similar results. A survey covering 73 respondents from 256 Norwegian law firms and interviews with 10 of the largest firms shows that there was a strong belief in the potential benefits of KM (Gottschalk 1999). Another survey covering 170 construction organizations (consultants and contractors) in the UK shows that about $40 \%$ already had a KM strategy, another $41 \%$ had plans to have a strategy within a year, and $19 \%$ did not have a strategy (Robinson et al. 2001). The research also found that about $50 \%$ of UK construction organizations had already appointed a Knowledge Manager or a special group with responsibility for implementing their KM strategy.

It is therefore agreed that $\mathrm{KM}$ is an important concept that attracts an increasing number of organizations and that those organizations that implement it first will gain more benefits. Tiwana (2000), believes that organizations which decide to wait until KM becomes a hot issue, are likely to fall behind, perhaps never to recover.

\section{$2.2 \mathrm{KM}$ in construction}

Although the term 'KM' is relatively new to construction organisations (Carrillo et al. 2000), many have adopted strategies for its implementation because the design and construction of projects 
involve several knowledge-intensive activities. These activities (e.g. preliminary design, analysis, detailed design, planning and managing the construction, maintenance, etc) are influenced by factors that are linked to human intelligence and knowledge such as experience and engineering judgment. The availability of knowledge is an important factor that affects construction projects as even experienced staff face difficulties when the required knowledge is not available and this can result in assumptions or judgments that may be disproved when knowledge becomes available (Kumar and Topping 1991).

Construction knowledge takes many forms e.g. experiences, best practices, lessons learnt, drawings, documents, etc. This knowledge is developed within design offices or on construction sites. Knowledge developed within a design office is easy to access by individuals or groups working within the same office but those located in geographically dispersed offices will not have the same ease of access to this knowledge. Knowledge generated on a construction site is rarely shared and this can result in loss of this knowledge. For example, if a problem relevant to the performance of a structure occurs on a construction site, engineers in the design office need to know about this problem; its nature, why it occurred and how it was solved so that they do not repeat mistakes in future designs. Improving the 'knowledge flow' within an organisation adds value, increases the ability to compete and helps to improve the quality of future projects.

Construction organisations can benefit from KM by implementing initiatives that help in capturing knowledge that is generated during the different stages of a project lifecycle to make it available and accessible in a timely fashion, throughout the organisation. Managing construction knowledge does not only contribute to increased safety and improved stability but also saves the time spent in design and construction and provides scope for innovation. Time can be saved, for example, by reducing the number of design cycles or by reducing the time used for searching for 
knowledge. Therefore, there is need for an approach that facilitates knowledge sharing within the construction industry.

\subsection{Barriers to KM implementation}

All the aforementioned surveys agree that organizations implementing KM face some barriers. The presence and strength of these barriers are dependent on many factors, such as the type of business processes, products, and clients. Construction organizations need to carefully investigate the barriers that are more relevant to construction activities in order to develop reliable KM strategies. Long lists of barriers are identified in the literature. However, they can be categorized into three main "barrier-groups": status of knowledge, location of knowledge, and culture surrounding the knowledge. Other barriers, such as high cost of KM systems, are not considered as main barriers because they are easier to address e.g. allocating a budget for implementing KM. The main barrier-groups are discussed in turn below.

\section{Status of knowledge}

Knowledge exists in a tacit or explicit status (Nonaka and Takeuchi 1995) and the allowance for an easy conversion from one status to another is important. Each status has its own characteristics, which may support or resist the conversion process. Knowledge, whether tacit or explicit, is either fully developed or still developing and this can also affect the conversion process as will be discussed below.

1. Tacit knowledge. This is stored in peoples' brains as mental models, experiences and skills and is difficult to communicate externally (Vail III 1999). The conversion of tacit knowledge takes two forms (Nonaka and Takeuchi 1995). It can be converted to another tacit knowledge through socialization in face-to-face interactions or to explicit knowledge through 
externalization by codifying an individual's knowledge. Capturing tacit knowledge and codifying it is one of the biggest challenges in KM (Bair \& O'Connor 1998). This knowledge, whether still 'developing' (in on-going projects) or already 'developed' (in previous projects), requires methodologies to help manage it wherever it exists. Capturing the 'developing' knowledge during the lifecycle of a project creates additional tasks in the congested agenda of employees. One of the evolving solutions is to integrate $\mathrm{KM}$ into the organizational processes. However, this could lead to several delays in finishing the work on time due to added activities. Furthermore, capturing the 'developed' tacit knowledge is far more difficult and requires advanced tools and strategies.

2. Explicit knowledge. This is encoded in organizational formal models, rules, documents, drawings, products, services, facilities, systems and processes and is easily communicated externally (Vail III 1999). Its conversion also takes two forms (Nonaka and Takeuchi 1995). It can be converted to tacit knowledge through internalization when an individual reads and understands well-coded knowledge. It can also be converted to another type of explicit knowledge through combining more than one form of knowledge to generate new knowledge. Although, conversion of explicit knowledge is easier than that of tacit knowledge, it still requires several resources such as time, technology, and commitment. Many organizations find that 'Developed' explicit knowledge is more difficult to manage than 'Developing' knowledge because it is hard to manage knowledge that was developed (in the past) by other individuals. In the long term, however, 'developed' knowledge is more critical to the organization's knowledge-base as it constitutes the memory of the organization. The difficulty associated with managing developed knowledge is with regards to allocating resources, especially time and people to find and store this knowledge. 


\section{Location of knowledge and its intended users}

Knowledge, whether tacit or explicit, exists in different sources and is required by different users. A source of knowledge can be a user of another knowledge, for example a person is a source when he contributes to a knowledge-base but he is a user when he reads from the knowledgebase. The transformation of knowledge from sources to users is facilitated or resisted by several factors. Understanding the relationships between these sources and users and their associated enablers and resistors is a difficult task requiring extensive examination. Moreover, it becomes more difficult to manage knowledge when its sources or users are geographically dispersed.

1. Geographically dispersed sources. In the construction context, these include: offices, employees, designers, contractors, suppliers, customers, and partners. The coordination of the process of capturing knowledge from geographically dispersed sources is obstructed by many factors. For example, capturing knowledge from people spending most of their time on construction sites is opposed by limited time and difficulty in getting technologies for capturing knowledge into the sites. Furthermore, having dispersed sources of knowledge (e.g. staff scattered in different countries) requires a central unit that plans and monitors the process to ensure that correct and valid knowledge is collected (Conheeney et al. 2000).

2. Geographically dispersed users. Organizations with geographically dispersed offices and employees need to ensure that the captured knowledge is safely delivered to users whenever they require it. Making this knowledge available to its intended users is faced by several challenges with regards to the methodology of transferring and sharing, supporting technology, and availability of time for accessing the transferred knowledge (Patel et al. 2000). 


\section{Culture}

Cultural barriers that obstruct the implementation of KM refer to the culture of individuals and organizational culture. Culture of individuals is about the way individuals act and respond. For example, not all employees in an organization will have the same willingness to share their knowledge. Organizational culture is more about the processes used within the organization. For example, some organizations consider review meeting as part of the design process and this encourages knowledge sharing. The cultural barriers that affect the implementation of KM are:

1. Willingness to share. "Why would I give my knowledge to others?" This is a typical question that is asked by people when they are asked to share their knowledge. Most of the literature focuses on the organizational benefits of KM neglecting the fact that tacit knowledge cannot be captured unless its holders realize that they also benefit (Scarborough et al. 1999). Employees need to understand that shared knowledge multiplies and that everyone needs knowledge from others. Secondly, people like to be rewarded for their contribution to the organizational knowledge-base and this necessitates the adoption of carefully developed reward systems that do not focus only on monetary figures but also include other schemes such as promotion, recognition, etc.

2. Availability of time. Employees find themselves under pressure of increased job tasks and delivery deadlines. Codifying tacit knowledge is difficult and time consuming and even storing and indexing codified knowledge is time intensive. Many organizations therefore find difficulties in allocating time to their staff to contribute their knowledge and to manage it. Employees also need time for training in order to understand the KM system so that they can use it efficiently. Employees who need to search for an answer to a question also find that they do not have enough time to search the knowledge-base. Instead, they normally prefer to ask an experienced colleague (Carrillo et al. 2000). 
3. Type and nature of business. Organizations differ according to the industry sector within which they perform. Some organizations have most of their work indoor, e.g. design offices while others have most of their work outdoors, e.g. civil engineering projects. Capturing knowledge from outdoor projects is very difficult due to two types of mobility. One is with regards to the formation of new teams for each project and the second is with regards to mobility of staff within the project site. Some project sites do not have a suitable environment for capturing knowledge or even for accessing a knowledge-base.

4. Technology infrastructure. Although technology is only a facilitator to $\mathrm{KM}$, many of the KM processes depend on it to allow for faster storage, retrieval, and transfer (Tiwana 2000). Technology therefore provides support for KM with a range of hardware and software tools. However, many organizations find it difficult to identify the tools that address their needs since this requires an understanding of the $\mathrm{KM}$ requirements for the organization and what these tools can offer. Using the wrong tools can result in a technology infrastructure that is not compatible with the existing technology within the organization or that does not address the organization's business goals.

5. Size of organization. The amount of knowledge available in an organization may be considered proportional to the size of the organization. Knowledge within a large organization is scattered throughout its offices and is therefore more difficult to manage. This necessitates that organizations identify what knowledge they need to manage and where it exists in order to achieve the organizational business goals (CIRIA 2000). This identification may not be a straightforward process as managing more than one knowledge type may be required. In this case, an organization needs to prioritize these types of knowledge based on the priorities of its business goals. 
6. Rewarding schemes may also create a barrier. Many KM initiatives focus on some kinds of rewarding schemes, especially short-term schemes. The problem with these schemes is that they consider rewarding people for what they do. This can create a new barrier because people may become more materialistic and will not accept new work unless they are explicitly rewarded for doing it. Therefore reward schemes need to be carefully designed so that they do not create a new barrier.

\subsection{Overcoming the barriers}

Literature and surveys show that many barriers obstruct the implementation of KM (Davenport 1997; KPMG 1998; Gottschalk 1999; TFPL 1999; Scarborough et al. 1999; Carrillo et al. 2000; CIRIA 2000; Patel et al. 2000; Storey and Barnet 2000; Tiwana 2000; Robinson et al. 2001). These barriers, if not properly addressed, can result in losing trust in the concept of KM (McConalogue 1999; Storey and Barnet 2000). New barriers can also result from poor practices or badly designed systems. Overcoming KM barriers is not an easy task and requires extensive planning (Al-Ghassani et al. 2001b). This necessitates fully understanding the nature of knowledge that needs to be managed and the barriers that resist its implementation. One of the best ways for understanding existing barriers is by having clear identification of KM problems at the early stages of developing strategies for KM (Al-Ghassani et al. 2000b). Early identification of $\mathrm{KM}$ problems is crucial because systems are more flexible during their design phase, while rectifying or altering a KM system at a later stage is difficult if at all possible, time-consuming, and expensive (CIRIA 2000). Organizations therefore need a tool that helps capture and define the KM problem so that reliable KM initiatives can be developed. Although numerous KM tools exist to support some elements of the KM process (Ruggles 1997; Jackson 1998; Bair \& O'Connor 1999; Tiwana 2000; Al-Ghassani et al. 2001a; Carrillo et al. 2000), no tool is found to facilitate understanding and defining the KM problem. 


\section{METHODOLOGY FOR IDENTIFYING KM PROBLEMS}

\subsection{Background}

Defining a KM problem requires intensive examination of several issues. A methodology for identifying KM problems is therefore developed within the CLEVER (마oss-sectoral Learning in the Virtual Enterprise) research project (Kamara et al. 2001). The CLEVER framework (Figure 2) is developed to support $\mathrm{KM}$ in construction and manufacturing organizations. It aims to clarify a vague $\mathrm{KM}$ problem into a set of specific $\mathrm{KM}$ issues, established within a business context in order to provide appropriate and relevant processes to solve the identified problems by:

- Defining the KM problem and linking it to business drivers/goals;

- Creating the desired characteristics of KM solutions;

- Identifying the critical migration paths to achieve the desired characteristics;

- Selecting appropriate KM processes to use on those paths; and

- Developing a strategy for implementing the selected KM processes.

$<$ Insert Figure 2 here $>$

The Framework addresses these objectives through the four main stages illustrated in Figure 2. The first stage, "Identify KM Problem", aims to clarify the overall KM problem within a business context to deliver a refined $\mathrm{KM}$ problem and a set of $\mathrm{KM}$ issues from the overall problem. The second stage, "Identify Current and Required KM Characteristics", introduces a series of knowledge dimensions e.g. explicit/tacit, critical/auxiliary, generic/project specific, etc. This stage asks users to identify certain characteristics about the knowledge that they are interested in managing. Following this, users are asked to identify 'existing' knowledge characteristics and the 'desired' knowledge characteristics. The third stage, "Identify Critical Knowledge Migration 
Paths", aims to allow users to select how best they would like to migrate from the 'existing' characteristics to the 'desired' characteristics i.e. either directly, or using intermediate steps. The last stage, "Select Generic KM Processes", aims to ensure that the organization is in a position to implement $\mathrm{KM}$ and helps in selecting the appropriate $\mathrm{KM}$ processes which, when tailored to a particular organization's need, will address the stated KM problem.

The subsequent sections discuss, in some detail, the methodology, development, and operation of a prototype developed to address the first stage "Identify KM Problem" through introducing the Problem Definition Template (PDT).

\subsection{The problem definition template}

The Problem Definition Template (PDT) represents a methodology developed for clarifying the overall KM problem within an organizational business context. It aims to assist users to 'think through' the problem in a 'structured way'. It therefore covers issues that can cause, if not properly addressed, a weak definition of KM problems. These issues are identified by AlGhassani et al. (2004) as: improper identification of the type and nature of knowledge that needs to be managed; unclear business goals from implementing KM initiatives; improper identification of the characteristics of knowledge; and poor understanding of the relationships between sources and users of knowledge and their associated enablers and resistors. In order to address these issues, the process consists of several stages, each comprising a set of investigations that address relevant KM issues. The developed approach allows organizations to:

- describe a 'vague' KM problem which does not need to be too specific at this stage;

- investigate the problem against the organizational business drivers;

- characterize the knowledge problem;

- identify the sources and users of the knowledge;

- identify the enablers and resistors for transferring the knowledge from sources to users; 
- $\quad$ link the knowledge problem to the relevant KM Process(es); and finally;

- refine the previously stated KM problem.

Using this approach allows organizations to identify if their business drivers have a KM dimension and enables them to develop strategies to address the KM problem. This new approach of gathering information for the identification of KM problems is simple to use and costeffective. It is applicable to large organizations as well as small-to-medium-sized organizations, which cannot afford the cost of external consultants. Figure 3 shows section of the PDT. <Insert Figure 3 here $>$

\subsection{Need for a software tool}

The PDT was tested by four industrial collaborators in individual workshops. The industrial collaborators included three construction organizations and one manufacturing organization. The workshops included directors, senior managers, and site personnel. The workshop participants were asked to complete a questionnaire with answers on a five-point scale. The questions covered the following issues:

- ease of use;

- layout;

- relevance to the business; and

- appropriateness of the explanation provided.

Based on the comments received from the workshops, it is evident that this approach provides a very useful way to structure thinking about KM problems. Furthermore, it was agreed that very little else exists to assist organizations in structuring their thinking in this way. However, to be truly useful to an organization, some modifications were suggested. Firstly, the paper-based format was seen as lengthy, uninviting and not easy to use without guidance. Participants thought that the guidelines included in the approach needed to be much condensed, simpler, 
and automated for the approach to be a readily usable tool. Secondly, it was felt that the worth of using the approach would be greatly enhanced by automating it through encapsulation in a software system to provide context sensitive help and remove the duplication of data entry. Without this, users could view its completion as a trivial exercise.

\section{DEVELOPMENT OF THE PROTOTYPE}

In order to address the comments received during the workshops, a prototype software system was developed. Several activities were involved in the development of the system including its system architecture, selecting the implementation environment and the final development.

\subsection{System Architecture}

The aim of the prototype is to simplify the use of the developed approach so that its format is more inviting and its use-time is reduced. To accomplish this aim, the system architecture, shown in Figure 4, was developed. The four stages provide a means for capturing the information required to define the KM problem. These stages are: type of knowledge; characteristics; sources and users; and current processes for managing knowledge. In the last stage, the user will be allowed to restate the KM problem and the answer will be fed back into the input of the first stage. The arrows linking the four stages to one another indicate that entry, storage, viewing, and editing of information, can be done forward or backward from any stage. The arrows linking the stages to 'information storage' show that the information, once inputted, is immediately stored and can also be edited at any time during the process.

<Insert Figure 4 here> 


\subsection{Implementation environment}

Relational databases help in avoiding many problems such as redundancy problems, multiplevalue problems, update anomalies, insertion anomalies, deletion anomalies, avoiding data loss, and maintaining relational integrity (Roman 1999). In the development of the prototype for identifying KM problems, a general-purpose database package (Microsoft Access for Windows version 2000) was chosen for implementation. The choice of Microsoft (MS) Access as the database system for the development was based on the following rationale (Barker and Barker 1997; Kamara and Anumba 2001):

- it is a Microsoft product and hence allows future integration with potential programming software products such as Visual Basic and Visual C++;

- it facilitates the automation of many tasks without the need for programming skills;

- it allows the import of information from other sources, enabling users to work and maintain existing data in other applications;

- it provides attractive and convenient means of entering and editing information through the use of forms; and

- it offers to view the information in various forms through the use of queries and filters.

The prototype was developed in MS Access by means of creating tables, forms, macros, queries, and reports. Four tables were developed each representing one of the four main stages of the system architecture. A welcoming form was developed along with another four forms, each enabling the input to one of the tables. Other forms were embedded within the main forms and activated by the use of command buttons to provide help on how to complete the forms. A summary of the desired characteristics of the prototype and the way in which they were achieved is shown in Table 1.

<Insert Table 1 here> 


\section{SYSTEM OPERATION}

When the program is started, a welcoming screen is displayed. The three buttons at the bottom of the form allow the user to completely exit the Access database, or to cancel this application and remain within Access, or to proceed to the first form for information input.

Information can be inputted by answering a set of questions on: knowledge type, characteristics, suppliers and users, enablers and resistors and the current processes of managing knowledge. Four forms are used to input, edit, and view information. While completing the forms, the user will have the opportunity to return to any previous form to modify the input. Once all forms are completed, the user can click the 'Restate the knowledge problem' command button. This displays the KM problem that was inputted at the beginning of the process and allows the user to edit it. Figure 5 shows a sample screen for entering, viewing, and editing information. The easyto-follow labels in addition to the 'help' buttons give enough guidance for completing the forms.

\section{$<$ Insert Figure 5 here $>$}

The prototype is able to produce a report on the KM problem. Clicking 'Report preview' in the last form generates the report, which contains a refined KM problem and specific KM issues. This report can also be used as a reference point for the organization when developing its KM strategy.

\section{SYSTEM EVALUATION}

This section describes the evaluation of the prototype developed. The evaluation was undertaken by four construction organisations including design offices and contractors. These organisations are different from those involved in the workshops used for testing the paperbased version of the PDT. The evaluations took place in the organisations' offices. Every evaluation consisted of three parts. First, a short introduction was given to the organization. 
Then, the participants from the organizations were allowed to use the prototype. The range of problems evaluated included 'risk management', 'transferring expertise' and 'acquiring knowledge in technical domains'. This was important in order to give the user a feel for the system. The author was seated next to the participants to provide guidance. This was followed by an open discussion after which participants were asked to complete a questionnaire. All participants were generally satisfied with the performance of the prototype and felt that it is useful for clarifying KM problems.

Response to the questionnaire shows that organizations were satisfied with the prototype developed. Questions were divided into three categories: KM Problem and Goals Identification, KM Strategy Development and General. A high average rating of 4 out of $5(80 \%)$ rated the prototype as effectively facilitating the clarification of KM problems in construction organisations. It was also agreed by the participants that the knowledge characteristics used are relevant to construction organisations as participants gave an average rating of 4.25 (85\%). Participants gave an average of $3.75(75 \%)$ on their satisfaction that the prototype properly relates construction knowledge to organisational business drivers. Participants also gave an average score of $3.75(75 \%)$ for the usefulness of the prototype in identifying the organisational goals from managing construction knowledge. Further comments received from the participants described prototype as: 'overall excellent product', 'very logical flow', and 'potentially very useful'.

\section{DISCUSSION}

The developed prototype introduces a new IT tool for identifying KM problems within a business context of organizations. The prototype is applicable to different types of organizations because it investigates the main aspects of $\mathrm{KM}$ and covers a wide range of $\mathrm{KM}$ issues. Flexibility was 
considered when designing the prototype so that users can add to the already built-in information. The system is simple to use, requires a relatively short time to complete, and contains a guide that provides help during each stage of its operation. The prototype is also equally applicable to strategic and tactical KM problems.

The rationale underpinning the development of this prototype is based on the fact that all existing approaches focus on the operational/implementation level of $\mathrm{KM}$ and that no tool is available to support KM at the strategic level (Al-Ghassani et al. 2001a). One of the first activities at the strategic level of $\mathrm{KM}$ is to define the problem as unclear problems or business goals can lead to losing trust in the concept of KM (Sorey and Barnet 2000; Tiwana 2000). What is required therefore, is a prototype that supports the understanding and clarifying of KM problems within the business context of an organization (Al-Ghassani et al. 2001a; Kamara et al. 2001). The prototype therefore linked the KM problem to the business drivers for change within an organization.

\section{CONCLUSIONS}

This paper provided an overview of knowledge management and its current practice in the different industries. It stated that a useful way for addressing existing barriers and for preventing new ones from evolving is by having clear identification of KM problems at the early stages of developing the KM strategy. It then presented the methodology, development, operation, and evaluation of a prototype software system developed to assist organizations in identifying their KM problems within a business context. The system is able to produce a report containing a summary of the KM problem and a distilled set of specific KM issues. This report forms an appropriate platform for the development of methods and strategies for KM in any business organization. The prototype was evaluated and it was agreed that it is a very useful way to 
structure thinking about KM problems. It was also found useful for both tactical and strategic KM problems for a wide range of organizations. The inviting format, simplified guidance, reduced input duplication, and automated report generation were found very potential for attracting organizations to use the system. The prototype has since been beta tested in two leading international organizations (one construction and one manufacturing) and has now been converted into a commercial product.

\section{REFERENCES}

Al-Ghassani, A.M., Carrillo, P.M., Anumba, C.J., and Robinson, H.R. (2001a). "Software Requirements for Knowledge Management in Construction Organisations", Proc., 17th Annual ARCOM Conf.., University of Salford, Salford, UK, 199-206.

Al-Ghassani, A.M., Kamara, J.M., Anumba, C.J., and Carrillo, P.M. (2001b). "An Innovative Tool for Knowledge Problem Definition", Proc., 1st Int. Conf. on Innovation, in AEC., , Loughborough University, UK, 249-256.

Al-Ghassani, A.M., Kamara, J.M., Anumba, C.J. and Carrillo, P.M. (2004), "An innovative approach to identifying knowledge management problems" , Engineering, Construction and Architectural Management , 11(5), 349-357.

Anumba, C.J., Carrillo, P.M., Backhouse C.J., Brookes, N.S., and Sinclair, M.A. (2001). "Crosssectoral Learning in the Virtual Enterprise (CLEVER)", EPSRC Final Report Appendix (on $C D)$, October, Loughborough University, UK.

Bair, J.H., and O'Connor, E. (1998). "The State of the Product in Knowledge Management", J. of Knowledge Management, 2 (2), 20-27.

Barker, F. S. And Barker, D. (1997). Using Access 97, QUE Corporation, USA.

Blumentritt, R., and Johnston, R. (1999). "Towards a strategy for Knowledge Management", Technology Analysis and Strategic Management, 11(3), 287-300. 
Britton, C., and Doake, J. (1996). Software System Development: a gentle introduction, London, McGraw-Hill.

Carrillo, P. M., Anumba, C.J., and Kamara, J.M. (2000). "Knowledge Management Strategy for Construction: Key I.T. and Contexual Issues", Proceedings International Conference on Construction Information Technology 2000., Reykjavik, Iceland, 1, 155-165

CIRIA (2000). Workshop Report on Applying Knowledge Management to the Construction Industry, Construction Productivity Network CPN E0100, London, UK.

Conheeney, K., Stephenson, P., and Griffiths, A. (2000). "Issues in knowledge transfer within a construction training and learning organisation", Proceedings International Conference on Construction Information Technology.2000., Reykjavik, Iceland, 1, 229-240.

Connell, J.L., and Shafer, L.I. (1995). Object-Oriented Rapid Prototyping, Yourdon Press, Prentice Hall Building, New Jersey.

Davenport, T. (1997). "Secrets of Successful Knowledge Management". Issue of Knowledge Inc., Feb, <http://webcom.com/quantera/secrets.html> 04/09/2000.

Davenport, T., and Prusak L. (1998). Working Knowledge: how organizations manage what they know, Harvard Business School Press, Boston, Massachusetts.

Gottschalk, P. (1999). "Use of IT for Knowledge Management in Law Firms", JILT, 3, <http://www.law.warwick.ac.uk/jilt/99-3/gottschalk.html> last viewed 10/12/2001.

Jackson, C. (1998). "Process to Product: creating tools for knowledge management", Proc. 2nd Int. Conf. on Technology Policy and Innovation, Assessment, Commercialisation and Application of Science and Technology and the Management of Knowledge, Lisbon, Portugal. Kamara, J.M., and Anumba, C.J. (2001). "ClientPro: a prototype software for client requirements processing in construction", Advances in Engineering Software, 32, 141-158. 
Kamara, J.M., Anumba, C.J., and Carrillo, P.M. (2001). "Selection of a Knowledge Management Startegy for Organisations", Proc. of the 2nd Euro. Conf. on Knowledge Management, Bled, Slovenia, 243-254.

Kanter, J. (1999). "Knowledge Management: Practically Speaking", Information Systems Management, 16(4), 7-15.

Kazi, A. S., Hannus, M., and Charoenngam, C. (1999). "An Exploration of Knowledge Management for construction", Proc. of 2nd Int. Conf. on CE in Construction, CIB Publication 236, Espoo, Finland, 247-256.

Klasson, K. (1999). "Managing Knowledge for Advantage: Content and Collaboration Technologies", The Cambridge Information Network J., 1(1), 33-41.

KPMG (1998). Knowledge Management Research Report, KPMG Management Consulting. Kummar, B., and Topping, B.H.V. (1991). Knowledge-based Processing for Structural Design, Proceedings of the Institute of Civil Engineers, 1(90), 421-446.

McConalogue, N.H. (1999). "Knowledge Management in the Construction Industry: A Study of Major UK Contractors", MSc Thesis, Dept. of Civil and Building Engineering. Loughborough University, Loughborough, UK.

Newman, B. D. (1991). "An Open Discussion of Knowledge Management", The Knowledge Management Forum, <http://www.3-cities.com/ bonewman/what_is.htm> 05/10/2001 Nonaka, I., and Takeuchi, H. (1995). The Knowledge Creating Company: How Japanese Companies Create the Dynamics of Innovation, Oxford University Press, New York, USA Patel, M. B., McCarthy, T. J., Morris, P.W.G., and Elhag, T.M.S. (2000). "The Role of IT in Capturing and Managing Knowledge for Organisational Learning on Construction Projects", Proceedings International Conference on Construction Information Technology 2000, Reykjavik, Iceland, 2, 674-685.

Robinson, H.S., Carrillo, P.M, Anumba, C.J., and Al-Ghassani, A.M. (2001). "Perception and Barriers in Implementing Knowledge Management Strategies in Large Construction 
Organisations", Proceedings of the Royal Institution of Chartered Surveyors COBRA Conference, Glasgow, United Kingdom, 451-460.

Roman, S. (1999). Access Database Design \& Programming, $2^{\text {nd }}$ edn, O'Reilly \& Associates, Inc., USA.

Ruggles, R. (1997). "Knowledge Management Tools: using technology to manage knowledge better", Working paper for "Ernst and Young", <http://www.businessinnovation.ey.com/mko/html/toolsrr.html> 26/8/2000

Scarbrough, H., Swan, J., and Preston, J. (1999). "Issues in People Management: Knowledge Management: a literature review", Institute of Personnel and Development, The Cromwell Press, Wiltshire.

Snowden, D. (1998). "A Framework for Creating a Sustainable Programme", Knowledge Management: a real business guide, S. Rock, ed., Caspian Publishing, London, 6-18.

Storey, J., and Barnet, E. (2000). "Knowledge Management Initiatives: learning from failure", J. of Knowledge Management, 4(2), 145-156.

TFPL Ltd. (1999). A report on: Skills for Knowledge Management - building a knowledge economy. $1^{\text {st }}$ edn, London.

Tiwana, A. (2000). The Knowledge Management Toolkit. Prentice Hall Inc., New Jersey.

Vail III, E. F. (1999). "Knowledge Mapping: Getting Started with Knowledge Management", Information Systems Management, 16(4), 16-23. 
Table 1. Summary of how Desired Characteristics of the Prototype were Achieved

\section{Desired Characteristics}

How Achieved

Allow convenient entry, viewing, and editing of information at any stage

Allow for any number of fields in a table

Support on how to complete the forms

Allow re-stating the problem

Facilitate the generation of a report on the problem

Allow for future integration with other KM frameworks and tools
The designed forms allowed the display and editing of stored data while command buttons inserted in the forms allowed easy navigation between the forms at any stage of the activity.

To overcome the limit of fields allowed in one table, four tables were developed with one-to-one relationships.

Whenever required, help buttons where created to provide information and instructions on how to complete the forms.

A command button, in the last form, allows for re-stating the $\mathrm{KM}$ problem, which is then fed back to the first form.

A report summarizing the $\mathrm{KM}$ problem was designed. $\mathrm{A}$ command button was used to open the report.

As an application of the MS Office group, "MS Access" can be easily linked to other packages. 


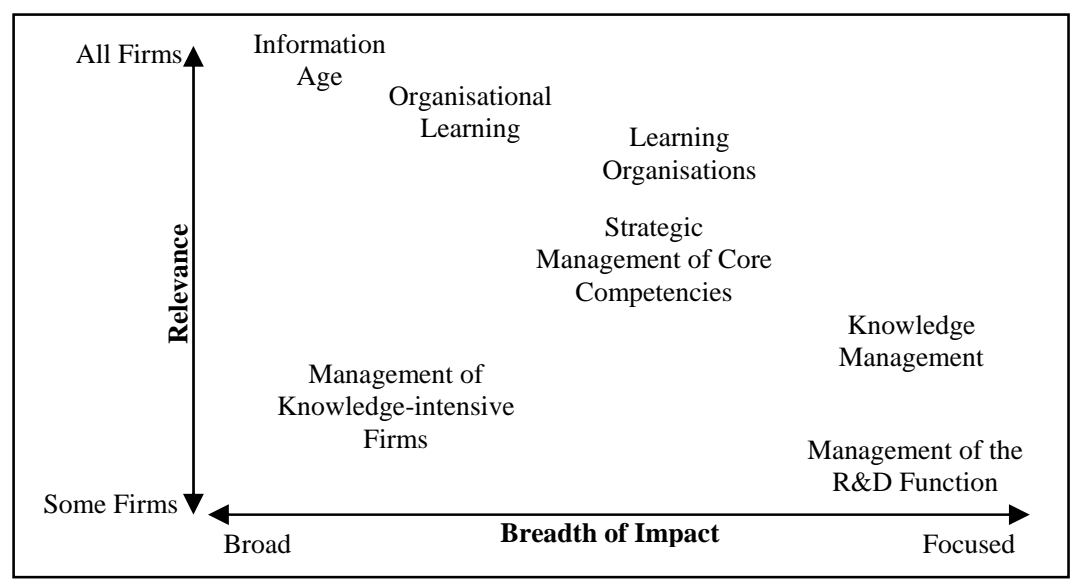

Figure 1: Relationship between KM and other concepts. Source: Scarborough et al. (1999) 


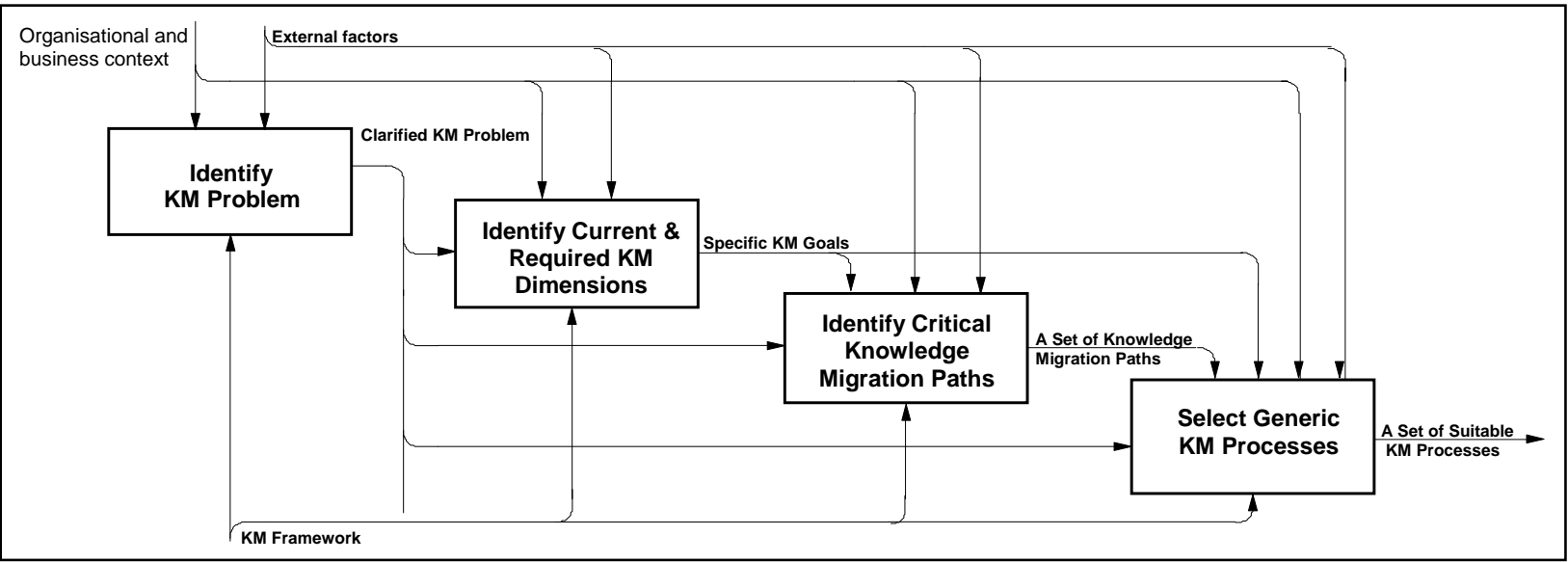

Figure 2: The CLEVER Framework for Knowledge Management 


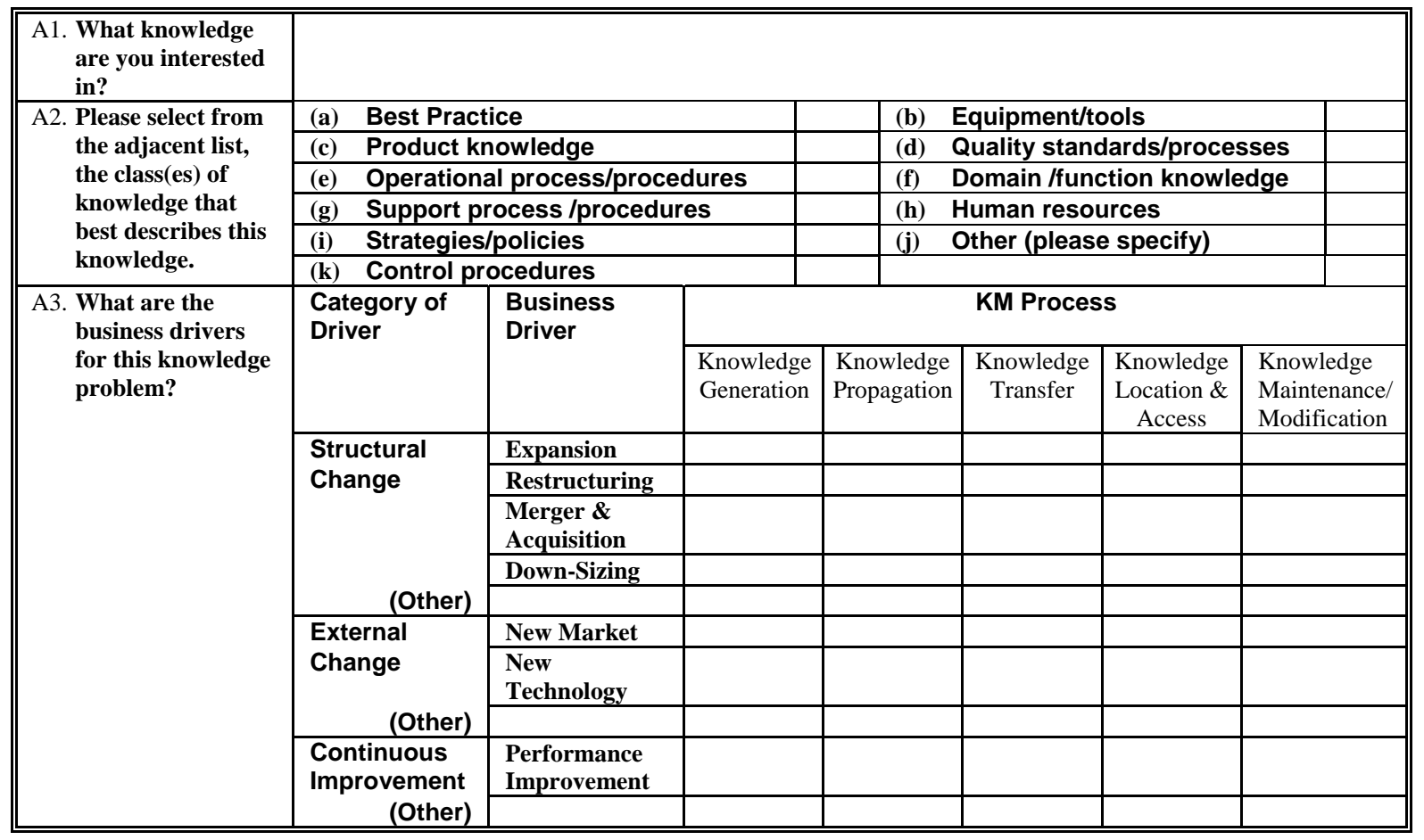

Figure 3: Section of the paper version of Problem Definition Template 


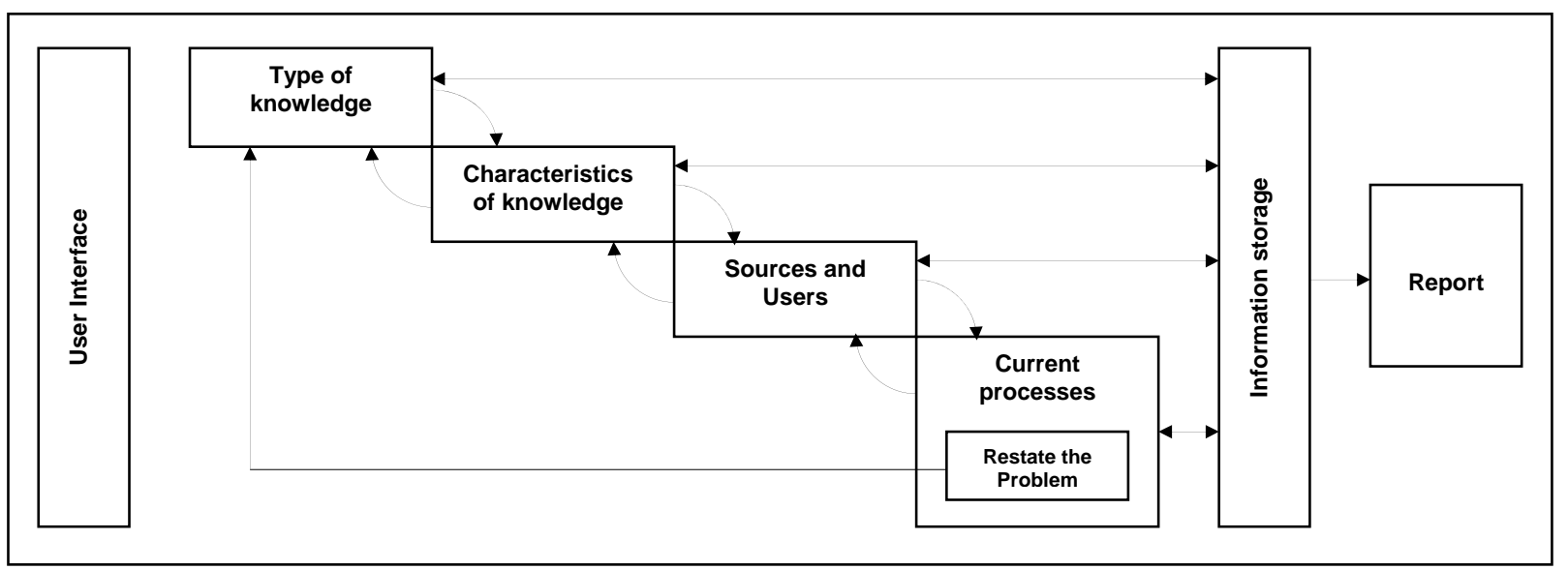

Figure 4: System Architecture of Problem Definition Template 


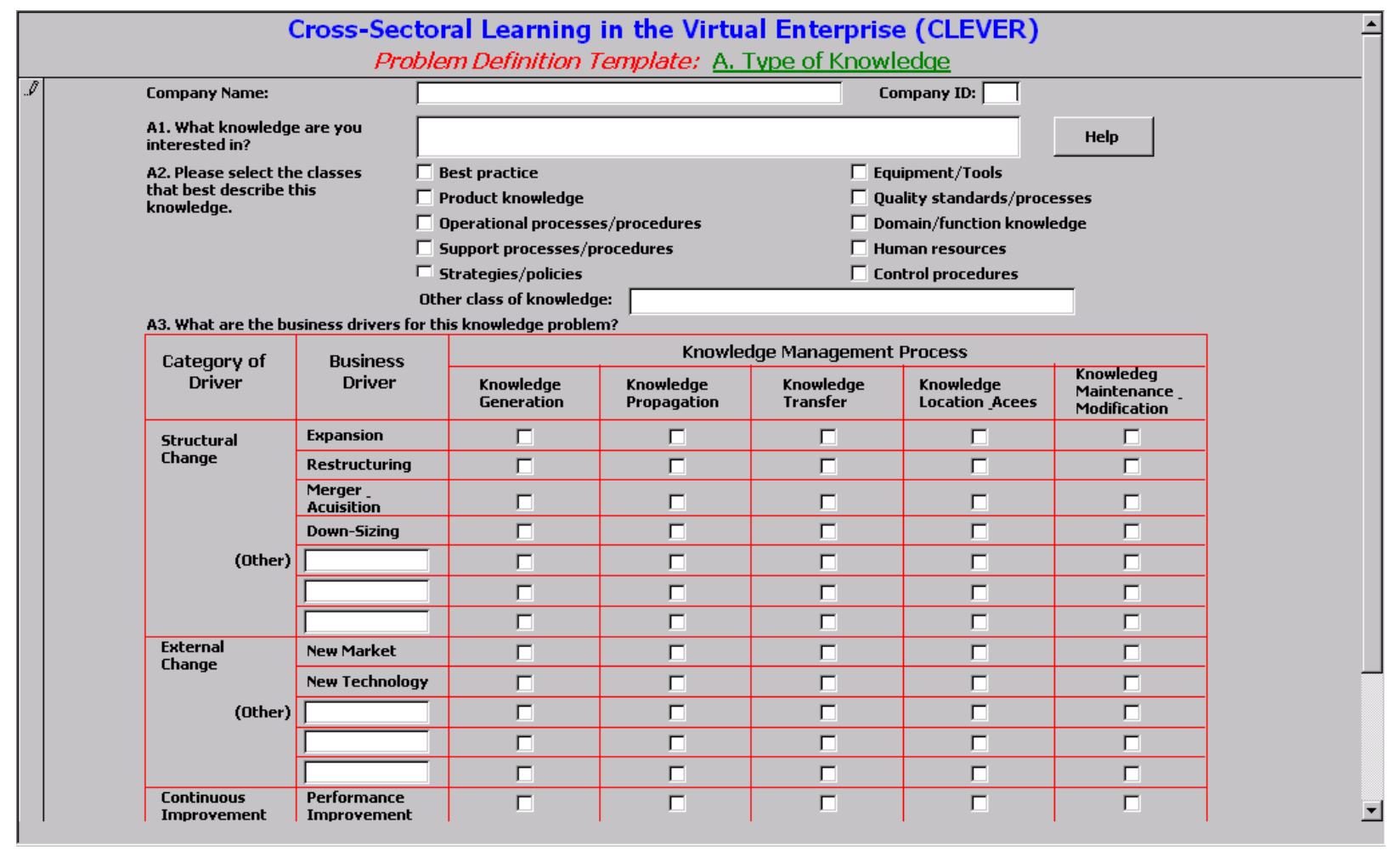

Figure 5: Sample screen for entering, viewing, and editing information 